\title{
Aligned Nanofiber Topography Directs the Tenogenic Differentiation of Mesenchymal Stem Cells
}

\author{
Tracee L. Popielarczyk ${ }^{1}$, Amrinder S. Nain ${ }^{2}$ and Jennifer G. Barrett ${ }^{1, *}$ \\ 1 Department of Large Animal Clinical Sciences, Marion duPont Scott Equine Medical Center, \\ Virginia-Maryland College of Veterinary Medicine, Virginia Tech, Leesburg, VA 20176, USA; popielat@vt.edu \\ 2 Department of Mechanical Engineering, College of Engineering, Virginia Tech, Blacksburg, VA 24061, USA; \\ nain@vt.edu \\ * Correspondence: jgbarrett@vt.edu; Tel.: +1-703-771-6856
}

Academic Editor: Daniel X.B. Chen

Received: 15 December 2016; Accepted: 23 December 2016; Published: 6 January 2017

\begin{abstract}
Tendon is commonly injured, heals slowly and poorly, and often suffers re-injury after healing. This is due to failure of tenocytes to effectively remodel tendon after injury to recapitulate normal architecture, resulting in poor mechanical properties. One strategy for improving the outcome is to use nanofiber scaffolds and mesenchymal stem cells (MSCs) to regenerate tendon. Various scaffold parameters are known to influence tenogenesis. We designed suspended and aligned nanofiber scaffolds with the hypothesis that this would promote tenogenesis when seeded with MSCs. Our aligned nanofibers were manufactured using the previously reported non-electrospinning Spinneret-based Tunable Engineered Parameters (STEP) technique. We compared parallel versus perpendicular nanofiber scaffolds with traditional flat monolayers and used cellular morphology, tendon marker gene expression, and collagen and glycosaminoglycan deposition as determinants for tendon differentiation. We report that compared with traditional control monolayers, MSCs grown on nanofibers were morphologically elongated with higher gene expression of tendon marker scleraxis and collagen type I, along with increased production of extracellular matrix components collagen $(p=0.0293)$ and glycosaminoglycan $(p=0.0038)$. Further study of MSCs in different topographical environments is needed to elucidate the complex molecular mechanisms involved in stem cell differentiation.
\end{abstract}

Keywords: tendon; differentiation; topography; scaffolds; nanofiber

\section{Introduction}

Tendon injury is a common clinical problem among veterinary and human patients with significant similarities in physiology and tendon pathologies. The inherent regenerative capacity of tendon is poor due to low cellularity and low vascularity of the tissue. These characteristics contribute to prolonged and incomplete healing with a high risk of re-injury [1]. Mesenchymal stem cells (MSCs) are used in tissue engineering and regenerative medicine applications for musculoskeletal tissue repair, particularly for their regenerative capabilities. MSCs can differentiate into tenocytes to promote tendon healing [2-4]. Engineered in vitro nanofiber scaffolds mimic the native topography of the extracellular matrix (ECM) microenvironmental cues that influence cell response and function including adhesion, migration, proliferation, and differentiation [5,6]. Scaffold directed MSC differentiation is determined through a combination of morphology, panels of tendon-related markers, collagen production and alignment, and levels of glycosaminoglycan (GAG) content $[7,8]$. Scleraxis (SCX), a transcription factor, is a key tendon-related marker which functions in tendon development and differentiation and promotes the expression and production of type I collagen (COL I) [9-11]. Type I collagen is the main 
fibrillar collagen functioning as a structural protein in healthy tendon composition [12]. GAGs aid in collagen fiber growth and function; abnormal levels signify disease or injury [13].

A challenge of designing scaffolds is determining the specific topographical cues of fiber diameter, orientation, and alignment that are most suited for tendon studies. Techniques used to manufacture fibrous scaffolds vary in their ability to control these parameters and produce various combinations. Fiber alignment influences MSC-fiber interaction and guides cell behavior. Kim et al. fabricated a nanofiber/microfiber composite scaffold that enhanced cell adhesion, cell spreading, and scaffold infiltration [14]. In another study, multilayered aligned electrospun scaffolds provided an anisotropic environment that promoted expression of tenomodulin, a marker of tendon maturation, and alignment of collagen throughout the scaffold in comparison to nonaligned scaffolds [15]. Yin et al. showed that compared to random electrospun fibers, aligned nanofibers provided microenvironmental cues for differentiation into the teno-lineage [16]. MSCs seeded on aligned nanofibers showed evidence that topography alone promoted changes in cell morphology and orientation. Cell differentiation was only enhanced by addition of TGF- $\beta 3$ [6] and printing of growth factors on aligned fibers [17]. These studies demonstrate that differentiation is a complex process sensitive to environmental cues, thus necessitating the need to optimize fibrous and growth factor parameters to mimic native environment to achieve specific lineages.

Electrospinning is a common manufacturing technique used to engineer fibrous scaffolds for tissue engineering. Improvements to this technique, including the use of modified collectors and fiber manipulation, have increased the applicability of this technique [18-21]. Besides electrospinning, other approaches including micropatterning, electro-chemically aligned have been used to study differentiation. For example, Gilchrist et al. studied the effects of architectural cues on aligned tissue formation using micropatterned aligned scaffolds of varying lengths and orientation and concluded that the combination of cues i.e. fiber length, boundaries, and orientation regulate tissue formation in a complex manner [22]. Kishore et al. investigated the effects of topography on tenogenesis with electro-chemically aligned threads, and showed alignment induced increased expression of scleraxis and tenomodulin [23]. In the context of fibrous scaffolds, the non-electrospinning Spinneret-based Tunable Engineered Parameters technique (STEP) [24] is a pseudo dry spinning method and a novel alternative to electrospinning, capable of depositing uniform nanofiber arrays. This method allows for the manufacturing of scaffolds with increased control of fiber diameter, spacing, length, alignment, orientation, and hierarchical assembly to influence cell response. The advantage of STEP fibers, in contrast to electrospun fibers, is that the parameter control allows for not only the traditional studies comparing aligned fibers to nonaligned or random fibers, but rather scaffolds of suspended and highly aligned fibers in different orientations [25].

Using STEP fibrous scaffolds, the aim of this study was to understand the biological response of MSCs to topographical cues provided by nanofibers to determine the optimal scaffold design to study tenogenesis. Polystyrene was used strictly for the purpose of studying MSC response to alignment and orientation. We hypothesized that MSCs would differentiate into tenocytes on scaffolds that mimic native tendon alignment specifically scaffolds of three-dimensional (3D) suspended and aligned nanofibers. MSCs from aligned parallel and aligned perpendicular nanofibers were compared to traditional two-dimensional (2D) monolayers with increases in gene expression of tendon-related genes, collagen production, and GAG levels. This data highlights specific cellular responses to alignment that adds to our understanding of stem cells in development and repair.

\section{Materials and Methods}

STEP scaffolds were manufactured as 3D suspended and aligned fibers in parallel and perpendicular orientation as described previously [24-26]. Nanofiber diameters were measured using environmental scanning electron microscopy. Equine bone marrow MSCs were seeded at a density of $2 \times 10^{5}$ cells per scaffold on a total of 154 scaffolds. In addition, MSCs were seeded on cell culture treated plastic for monolayer controls. Microscopy, qPCR, and biochemical data are in 
reference to monolayer. Experiments were performed in triplicate. Gene expression analysis of SCX and COL I was assessed on day 7 and day 14 from scaffolds relative to monolayers. Production of soluble collagen and GAG content in the media were assessed on days 1, 7, and 14 and averaged from 6 scaffolds per time point for collagen production and averaged from 8 scaffolds per time point for GAG content.

\subsection{Scaffold Manufacturing and Characterization}

Fibers were spun on scaffolds using the STEP technique (US9029149 B2 patent) to manufacture multi-layered, aligned suspended nanofibers in parallel or perpendicular orientations [24-26]. Briefly, the polymer is pushed through a syringe pump and stretched out into a continuous fiber as the spinneret comes in contact with the rotating frame and the solvent evaporates. Fiber surface area and density are determined by a combination of factors; scaffold frame dimensions, polymer solution, and spinning parameters. Scaffolds were manufactured with dimensions measuring $4 \mathrm{~mm}$ in length $\times 4 \mathrm{~mm}$ in width. Standard polystyrene (Mw 1,571,000 and Mw 2,257,000 g/mol, Polymer Scientific Products, Ontario, NY, USA) solutions were prepared with xylene to produce $400 \mathrm{~nm}$ diameter fibers. All scaffolds were sterilized with $70 \%$ alcohol and plasma-treated with a high frequency generator (Electro-technic Products Inc., Chicago, IL, USA) before coating with $20 \mu \mathrm{g} / \mathrm{mL}$ of bovine fibronectin protein (Thermo Fisher Scientific, Waltham, MA, USA).

\subsection{Environmental Scanning Electron Microscopy}

Fibers from each batch of scaffolds were sputter-coated in gold/palladium alloy and imaged with a Quanta 600 FEG Environmental Scanning Electron Microscope (FEI, Hillsboro, OR, USA). Scaffolds were randomly selected from each batch and diameters of 25 fibers per scaffold were measured with ImageJ and Fiji plugin (National Institutes of Health [27]) from ESEM images.

\subsection{Isolation and Characterization of Primary Equine Mesenchymal Stem Cells}

MSCs were isolated from bone marrow aspirate harvested from the sternum of a one year old male horse, euthanized for unrelated conditions, following approval of the Institutional Animal Care and Use Committee (IACUC) of the Virginia Polytechnic Institute and State University and assessed via previously described techniques [28]. Briefly, bone marrow was collected, centrifuged to remove the plasma, and cultured in media containing low-glucose GlutaMAX DMEM with $110 \mathrm{mg} / \mathrm{mL}$ sodium pyruvate (Thermo Fisher Scientific, Waltham, MA, USA), supplemented with $10 \%$ MSCs fetal bovine serum (Thermo Fisher Scientific, Waltham, MA, USA), and 1\% sodium penicillin and streptomycin sulfate (Sigma, St. Louis, MO, USA) at $37^{\circ} \mathrm{C}, 5 \% \mathrm{CO}^{2}$ and $90 \%$ humidity. Media was added after two days, cells were fed on day 4 , washed in PBS on day 5 , and then fed every two days until cells were $80 \%$ confluent. Flow cytometry results showed $>99 \%$ of cells were positive for both markers of stemness OCT4 and CD90, 22\% positive for CD44, 19\% positive for MHC I, and non-detectable for MHC II. Multilineage potential of MSCs was confirmed through adipogenesis, chondrogenesis, and osteogenesis assays.

\subsection{Cell Culture and Scaffold Seeding}

Scaffolds were each seeded with $2 \times 10^{5}$ equine bone marrow MSCs and cultured for 14 days in 12 well plates. Scaffolds were transferred to new 12 well plates with new cell culture media $24 \mathrm{~h}$ after seeding. In addition, MSCs were seeded on plasma-treated, fibronectin-coated plastic as previously described for nanofibers for monolayer controls. All MSCs were passage 3. Culture media contained low-glucose GlutaMAX DMEM with $110 \mathrm{mg} / \mathrm{mL}$ sodium pyruvate (Thermo Fisher Scientific, Waltham, MA, USA), supplemented with 10\% MSCs fetal bovine serum (Thermo Fisher Scientific, Waltham, MA, USA), and $1 \%$ sodium penicillin and streptomycin sulfate (Sigma, St. Louis, MO, USA) at $37{ }^{\circ} \mathrm{C}, 5 \%$ $\mathrm{CO}^{2}$ and $90 \%$ humidity. 


\subsection{Phase-Contrast and Time-Lapse Microscopy}

Cell morphology was observed for days 0-14 under phase microscopy. Each time-lapse video was recorded for MSCs on scaffolds over a 12-h period. Scaffolds were seeded in $35 \mathrm{~mm}$ glass petri dishes and loaded into the on-stage incubator (Okolab, Naples, Italy) at $37{ }^{\circ} \mathrm{C}, 5 \% \mathrm{CO}^{2}$ and $90 \%$ humidity. Microscopy was performed using an AMG EVOS fl digital inverted fluorescence microscope (Thermo Fisher Scientific, Walham, MA, USA).

\subsection{Fluorescence Microscopy}

Seeded parallel and perpendicular oriented fibrous scaffolds were stained with calcein AM on day 14 to assess cell viability on polystyrene fibers. Scaffolds were washed in PBS and stained with calcein AM in low-glucose GlutaMAX DMEM for $30 \mathrm{~min}$ and washed in PBS. Seeded scaffolds were fixed in $4 \%$ paraformaldehyde, incubated with $0.2 \%$ Triton X-100 and stained with rhodamine phalloidin (Thermo Fisher Scientific, Walham, MA, USA), followed by Prolong Gold Antifade Mountant with DAPI (4',6-diamidino-2-phenylindole) (Thermo Fisher Scientific, Walham, MA, USA) for F-actin and nuclei staining, respectively. Microscopy was performed using an AMG EVOS fl digital inverted fluorescence microscope (Thermo Fisher Scientific, Waltham, MA, USA).

\subsection{RNA Isolation, Quantification, and Quantitative PCR}

MSCs were harvested from scaffolds, monolayers from tissue culture treated plastic, and tenocytes with TRIzol (Thermo Fisher Scientific, Walham, MA, USA). Parallel and perpendicular scaffolds were pooled into their respective groups on day 7 and day 14. An RNeasy Mini Kit (Qiagen, Hilden, Germany) was used to isolate the RNA. RNA quantification was performed using a Quant-iT Ribogreen RNA Assay Kit (Thermo Fisher Scientific, Walham, MA, USA). RNA was converted to cDNA using a high frequency RNA-to cDNA Kit (Thermo Fisher Scientific, Walham, MA, USA) and Eppendorf Thermal Cycler (Brinkmann Instruments Inc., Westbury, NY, USA). Applied Biosystems Real-Time 7500 PCR System (Thermo Fisher Scientific, Waltham, MA, USA) was used to run quantitative PCR (qPCR) with TaqMan Master Mix (Thermo Fisher Scientific, Waltham, MA, USA) and 20 ng of cDNA per $50 \mu \mathrm{L}$ PCR reaction. Relative quantitation was calculated using the Comparative $C_{T}$ method $\left(2^{-\Delta \Delta C T}\right)$. Gene expression was normalized to monolayer controls using Glyceraldehyde 3-phosphate dehydrogenase (GAPDH). Custom equine primer and probe sequences for GAPDH, scleraxis, and type I collagen were purchased (Thermo Fisher Scientific, Waltham, MA, USA).

\subsection{Biochemical Assay of Media Content}

Biochemical analyses of collagen and glycosaminoglycan (GAG) content were used to determine ECM production over 7 and 14 days. Solubilized collagen was measured using the Sircol Soluble Collagen Assay (Biocolor Ltd., Carrickfergus, Ireland, UK). Sulfated glycosaminoglycan content was measured using the 1,9-dimethylmethylene blue (DMMB) assay, referencing bovine chondroitin sulfate A [29]. All absorbance readings were measured at $540 \mathrm{~nm}$ with a HIDEX Chameleon V microplate reader (HIDEX, Turku, Finland).

\subsection{Statistical Analysis}

Two-way analysis of variance (ANOVA) was used to determine fiber orientation and temporal effects on gene expression and matrix production, followed by post hoc Tukey HSD (JMP Pro 10 Software, SAS Institute Inc., Rockville, MD, USA, 2015). Mean \pm standard error for each statistic was calculated for each scaffold design. $p$-value $\leq 0.05$ were considered significant. 


\section{Results}

\subsection{STEP-Manufactured Fibers Exhibit Fibril-Like Organization}

STEP scaffolds were manufactured as nanofibers in aligned parallel and aligned perpendicular orientation, suspended from a scaffold frame (Figure 1A). Environmental Scanning Electron Microscopy (ESEM) images of parallel (Figure 1Bi) and perpendicular (Figure 1Bii) oriented fibrous scaffolds show alignment and orientation of fibers. Fiber diameters measured $400 \mathrm{~nm} \pm 128.17 \mathrm{~nm}$ standard deviation (Figure 1C). STEP fibers provided a three-dimensional, multilayer, aligned structure with fibers comparable to the diameter range of 50-500 $\mathrm{nm}$ of native equine tendon collagen fibrils [30]. These scaffold designs enabled complete integration of cells into the scaffolds and provided increased cell to fiber interaction.

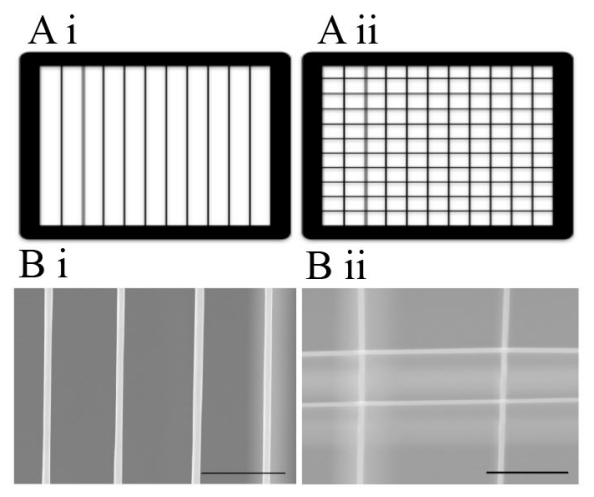

$\mathrm{C}$

\section{Suspended Fiber Diameters}

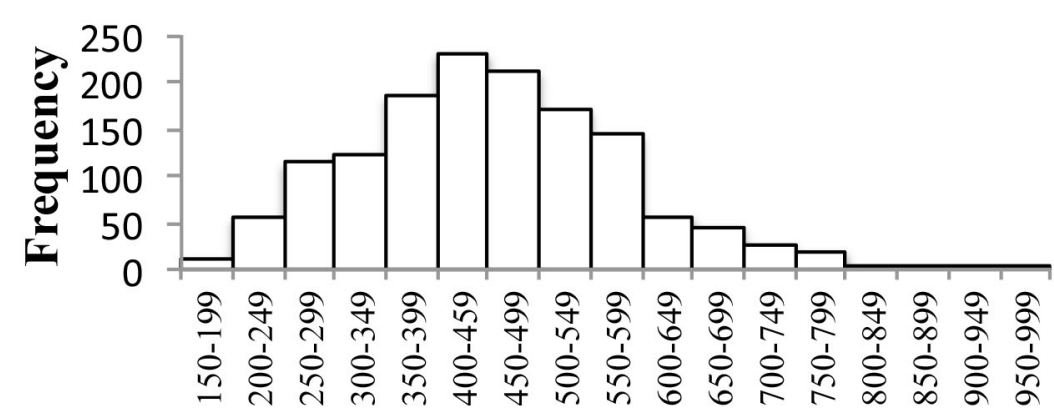

Fiber Diameter (nm)

Figure 1. STEP scaffold characterization of fiber diameter. A schematic of an aligned parallel fibrous scaffold (Ai) and an aligned perpendicular fibrous scaffold (Aii); SEM images of aligned parallel fibrous scaffold (Bi) and aligned perpendicular fibrous scaffold (Bii); Scale bar $5 \mu \mathrm{m}$ and $3 \mu \mathrm{m}$, respectively; Histogram of aligned parallel and aligned perpendicular fiber diameters, measured with ImageJ, $n=1400(\mathbf{C})$.

\subsection{MSCs Exhibit Tenocyte-Like Morphology on Suspended Fibrous Scaffolds}

MSCs were guided by the three dimensional structure of the fibers and fiber orientation as assessed by phase-contrast microscopy (Figure 2). MSCs on parallel fibers elongated along the nanofibers in a tenocyte-like morphology within $24 \mathrm{~h}$ of seeding (Figure 2A) and maintained by day 7 (Figure 2B). Morphological changes were similar on day 14. Parallel orientation of fibers promoted an elongated tenocyte-like morphology and maintained parallel MSC orientation between adjacent cells. Similar spindle-like MSC morphology was observed on perpendicular fibrous scaffolds. Additional cell morphologies were observed on perpendicular suspended nanofibers as MSCs spread across multiple fibers at intersections of fibers, on day 1 (Figure 2C) and day 7 (Figure 2D). 
Day 1
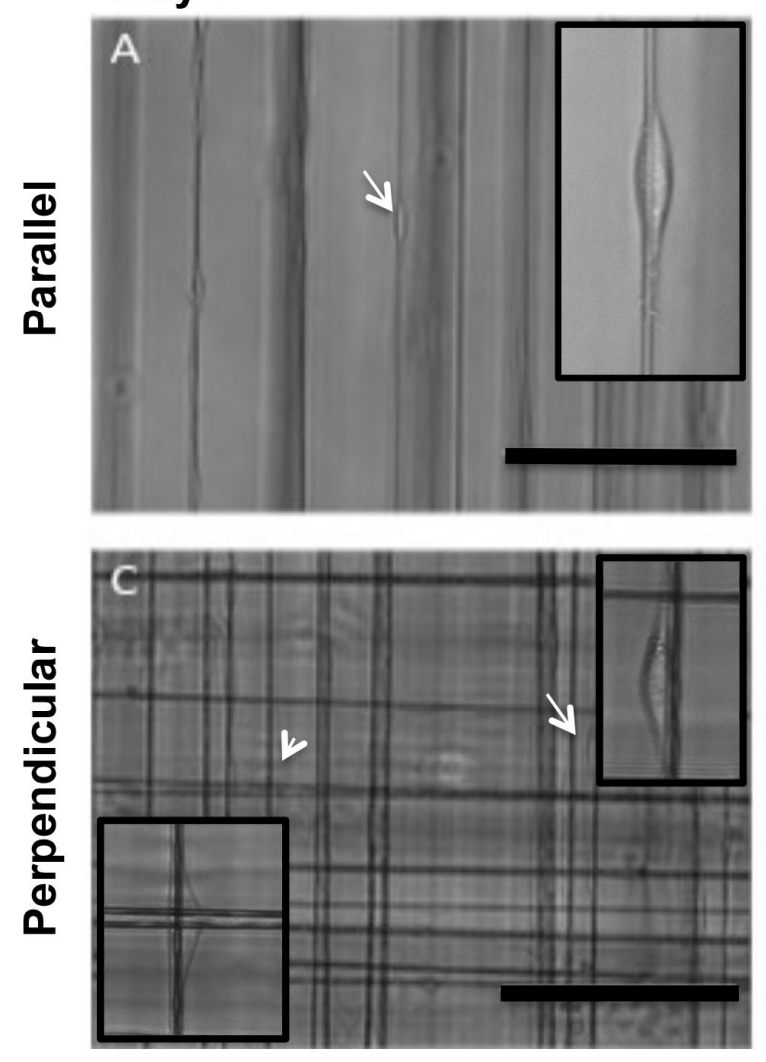

Day 7
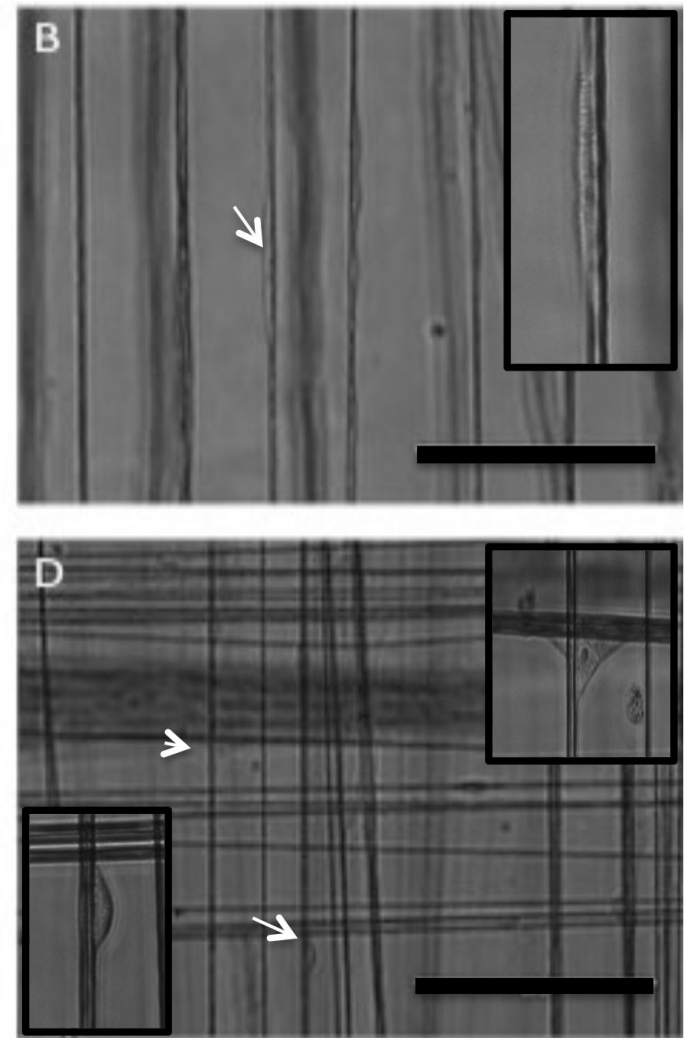

Figure 2. MSC morphology on aligned scaffolds. Representative phase-contrast microscopy images of equine bone marrow MSCs (mesenchymal stem cells) on aligned parallel oriented fibrous scaffolds $(\mathbf{A}, \mathbf{B})$ and aligned perpendicular fibrous scaffolds (C,D) on day 1 and day 7 of culture. Arrows, elongated cells; arrowheads, cell spreading. Insets show enlarged image of the cell. Scale bar $200 \mu \mathrm{m}$.

Cell migration and associated cell shape were better observed under time-lapse microscopy where we could clearly observe the cell-fiber interaction and changes in cell morphology (Figure 3). Time-lapse microscopy recording was started $24 \mathrm{~h}$ after seeding for parallel fibers (Video S1: Mesenchymal stem cell migration on aligned parallel nanofibers) and perpendicular fibers (Video S2: Mesenchymal stem cell migration on aligned perpendicular nanofibers). At this time, cell elongation was observed as a spindle-like morphology on parallel orientation of fibers and maintained MSC spacing between cells on adjacent fibers (Figure 3A-D). Perpendicular orientation of fibers allowed cells to migrate and spread along multiple fibers, temporarily change morphology, and switch directions at fiber intersections (Figure 3E-H).

Fibers of perpendicular orientation promoted changes in cell migration direction and cell morphology through interaction with intersections and spreading along multiple fibers. The elongated tenocyte-like morphology was predominant before and after cells migrated through the intersections. MSC monolayers showed typical polygonal morphology on standard tissue culture treated plastic (data not shown). Viability was maintained throughout 14 days of culture on both parallel (Figure 4A) and perpendicular fibers (Figure 4B) showing no toxicity of cells on the polystyrene nanofibers. 


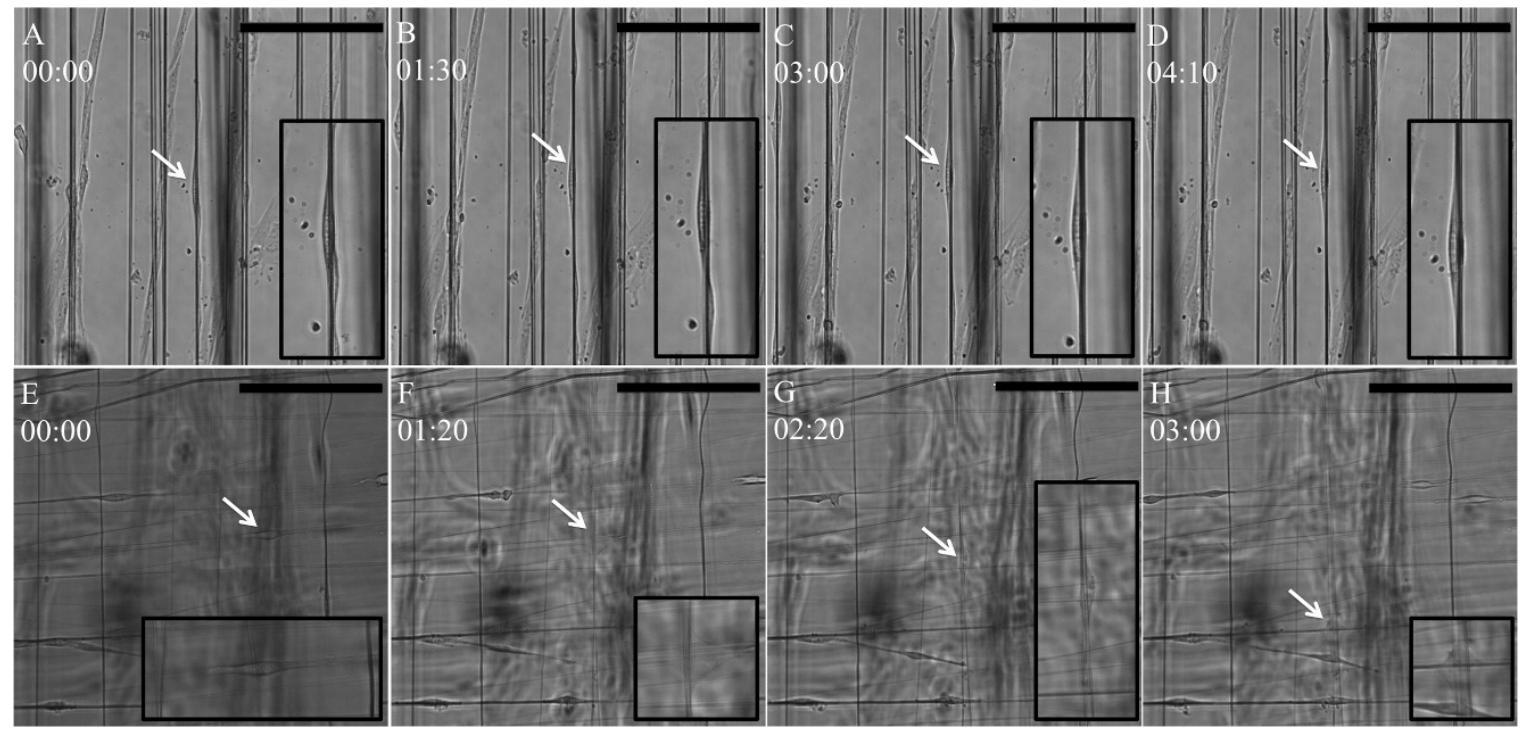

Figure 3. Time-lapse frames of MSCs on aligned fibers. Representative time-lapse microscopy images of equine bone marrow MSCs on aligned parallel fibrous scaffolds (A-D) and aligned perpendicular scaffolds (E-H) over $12 \mathrm{~h}$. Time (hours) is indicated in the upper left corner. Arrows tack cell migration during the time-lapse. Insets show enlarged image of the cell. Scale bar $200 \mu \mathrm{m}$.
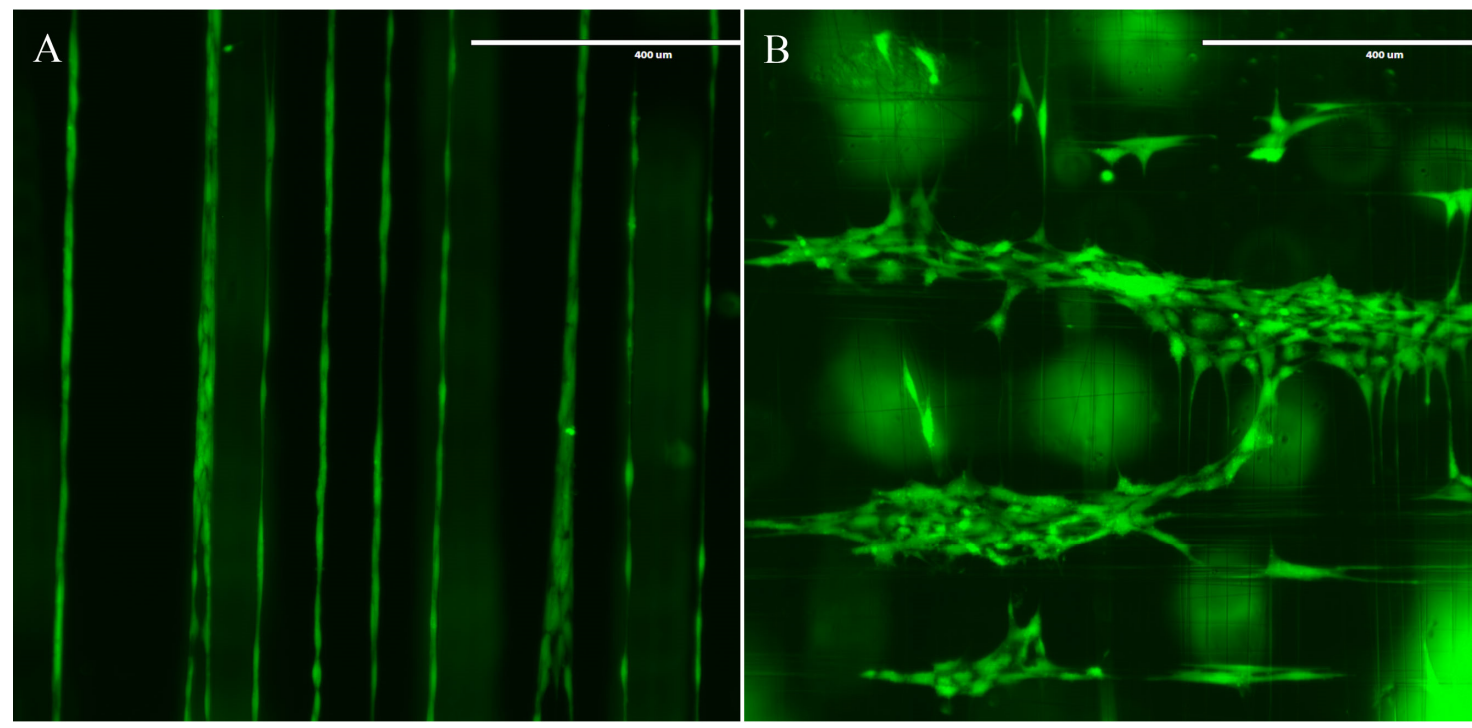

Figure 4. Viability of MSCs on aligned scaffolds. MSCs stained with calcein AM on parallel scaffolds (A) and perpendicular scaffolds (B) after 14 days of culture. Scale bar $400 \mu \mathrm{m}$.

MSCs cultured for 12 days on fibrous scaffolds elongated along aligned parallel fibers in both parallel and perpendicular oriented fibrous scaffolds as observed by fluorescence microscopy (Figure 5). Intracellular F-actin stained with rhodamine phalloidin and nuclei stained with DAPI were observed along the nanofibers and in the monolayers, showed cell attachment and interaction with fibers. Perpendicular fibrous scaffolds provided fiber intersections for cell interaction (Figure 5C,D), and parallel scaffolds maintained MSC spacing between fibers (Figure 5A,B) leading to parallel cell orientation and spacing between fibers. The above methods have shown that fiber alignment provides environmental cues, resulting in cell elongation/tenocyte-like morphology. 


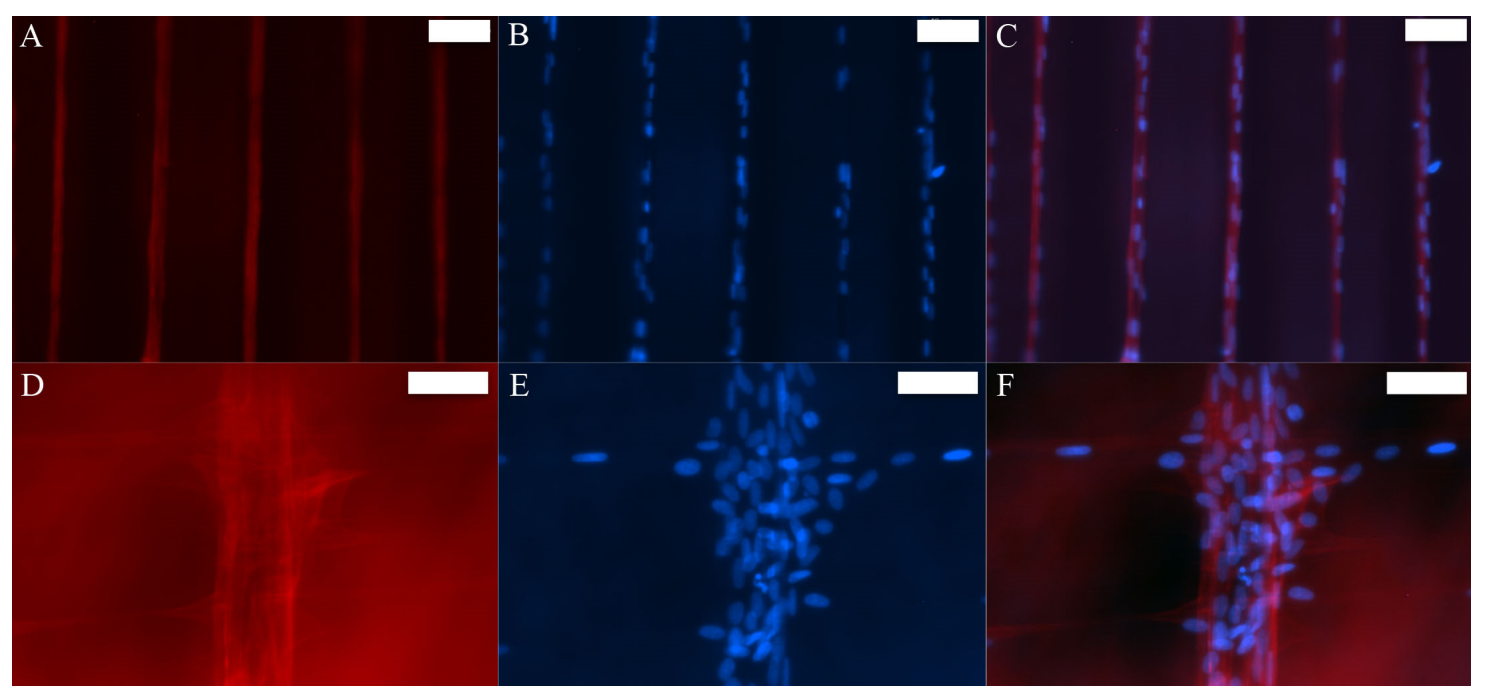

Figure 5. MSCs cytoskeleton and nuclei morphology on aligned scaffolds. Fluorescent microscopy images of equine bone marrow MSCs on an aligned parallel scaffolds $(\mathbf{A}-\mathbf{C})$ and an aligned perpendicular scaffolds (D-F) stained with rhodamine phalloidin (A,D); DAPI (B,E); and the merged image $(\mathbf{C}, \mathbf{F})$ on day 12 of cell culture. Scale bar $40 \mu \mathrm{m}$.

\subsection{Tendon-Associated Gene Expression Increased over Time}

Gene expression of SCX and COL I custom primers and probes (Table 1) was analyzed on day 7 and day 14 for monolayer and scaffolds to assess tenogenesis (Figure 6). There was an increasing trend in SCX expression between day 7 and 14, regardless of fiber orientation (Figure 6A). Similarly, expression of COL I increased between day 7 and 14, regardless of fiber orientation (Figure 6B). This combined increase of both SCX and COL I expression may provide evidence of early tenogenesis.

Table 1. Primer and probe sequences of tendon-associated genes for qPCR analysis.

\begin{tabular}{cccc}
\hline Transcript & Forward Sequence $\left(\mathbf{5}^{\prime} \mathbf{-} \mathbf{3}^{\prime} \mathbf{)}\right.$ & Reverse Sequence $\left(\mathbf{5}^{\prime} \mathbf{- 3}^{\prime} \mathbf{)}\right.$ & Probe Sequence $\left.\mathbf{( 5}^{\prime} \mathbf{- \mathbf { 3 } ^ { \prime }}\right)$ \\
\hline GAPDH & CAAGTTCCATGGCACAGTCAAG & GGCCTTTCCGTTGATGACAA & CCGAGCACGGGAAG \\
Scleraxis & CGCCCAGCCCAAACAG & TTGCTCAACTTTCTCTGGTTGCT & TCTGCACCTTCTGCC \\
Type I Collagen & GCCAAGAAGAAGGCCAAGAA & TGAGGCCGTCCTGTATGC & ACATCCCAGCAGTCACCT \\
\hline
\end{tabular}

A

SCX

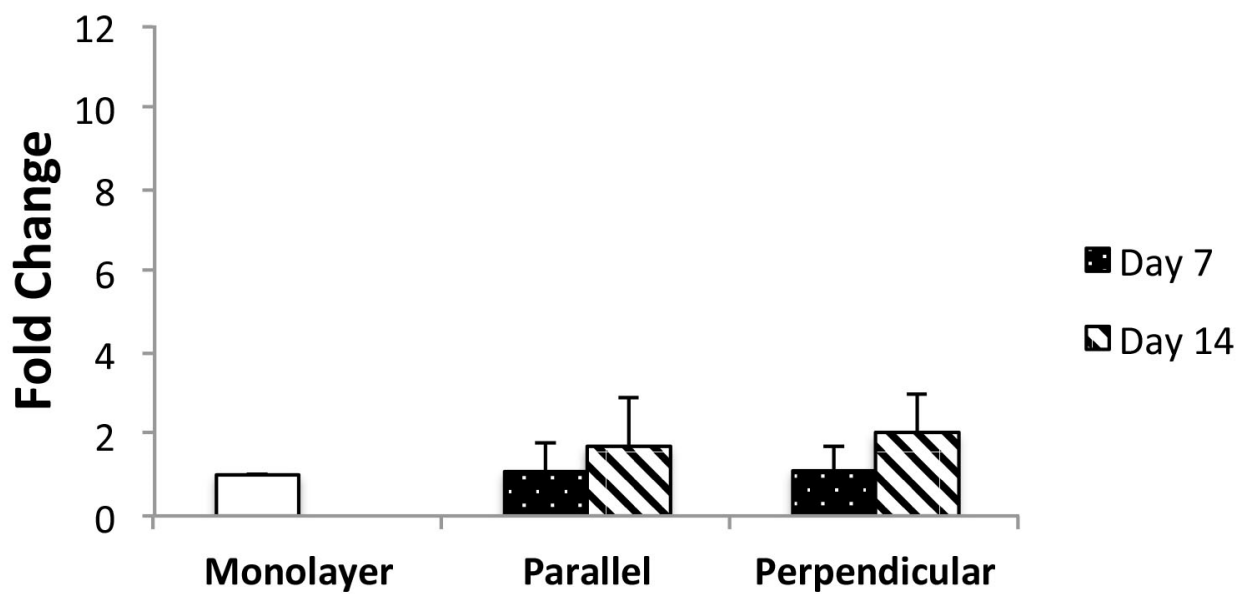

Figure 6. Cont. 
B COL I

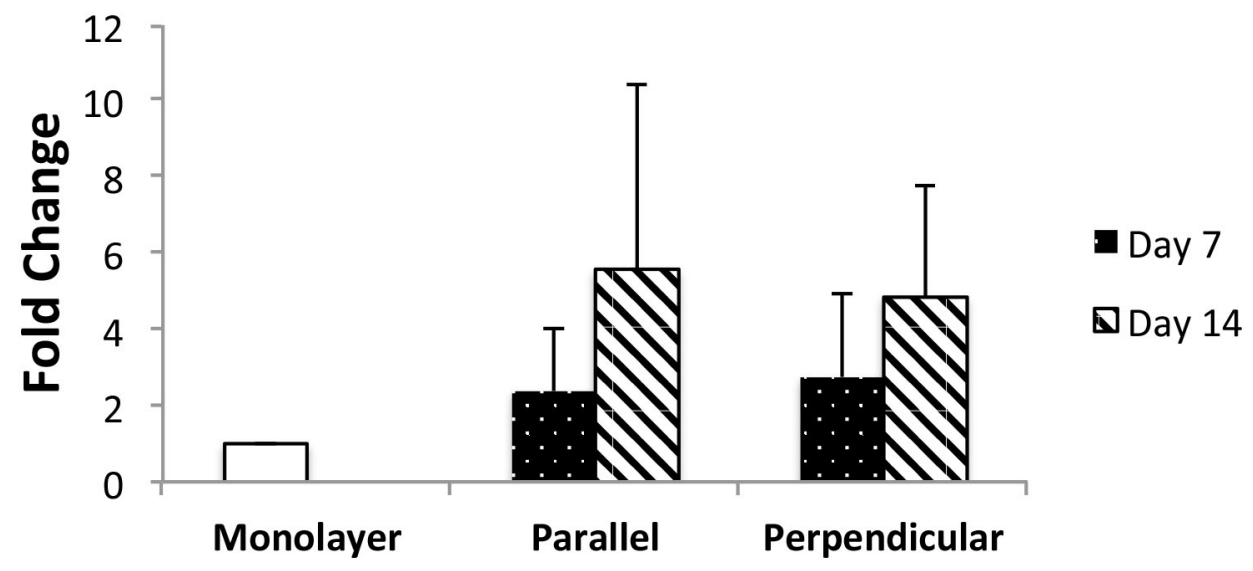

Figure 6. qPCR of MSCs from aligned scaffolds. Gene expression analysis of scleraxis (A) and type I collagen (B) from equine bone marrow MSCs collected from monolayers, aligned parallel scaffolds, and aligned perpendicular scaffolds on days 7 and 14. Genes were normalized to GAPDH (Glyceraldehyde 3-phosphate dehydrogenase). Gene expression of MSCs from scaffold designs is relative to monolayer controls. $p$-value $\leq 0.05$ were considered significant. Error bars represent SEM.

\subsection{ECM Production Increased over Time}

Extracellular matrix production was evaluated through biochemical analysis of collagen and GAG content in the media. We found the average collagen content to increase $(p=0.0293)$ between days 1, 7, and 14, regardless of fiber orientation (Figure 7A). We also found the GAG deposition to increase over time in all three groups (monolayer, parallel, and perpendicular). There was an increase in GAG production between days $(p<0.0001)$ and an increase between all days and fiber orientation $(p=0.0038)$. In addition to the gene expression data, this increase in matrix deposition is indicative of tendon development.

A

\section{Collagen Content}

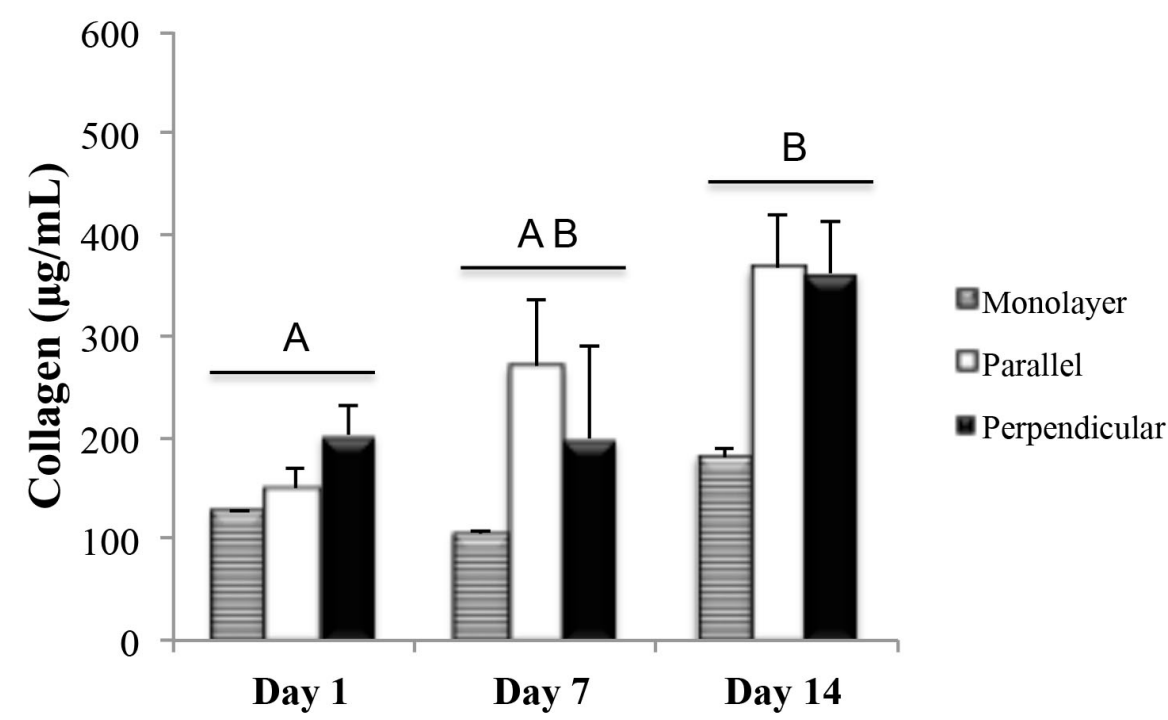

Figure 7. Cont. 


\section{B Glycosaminoglycan Content}

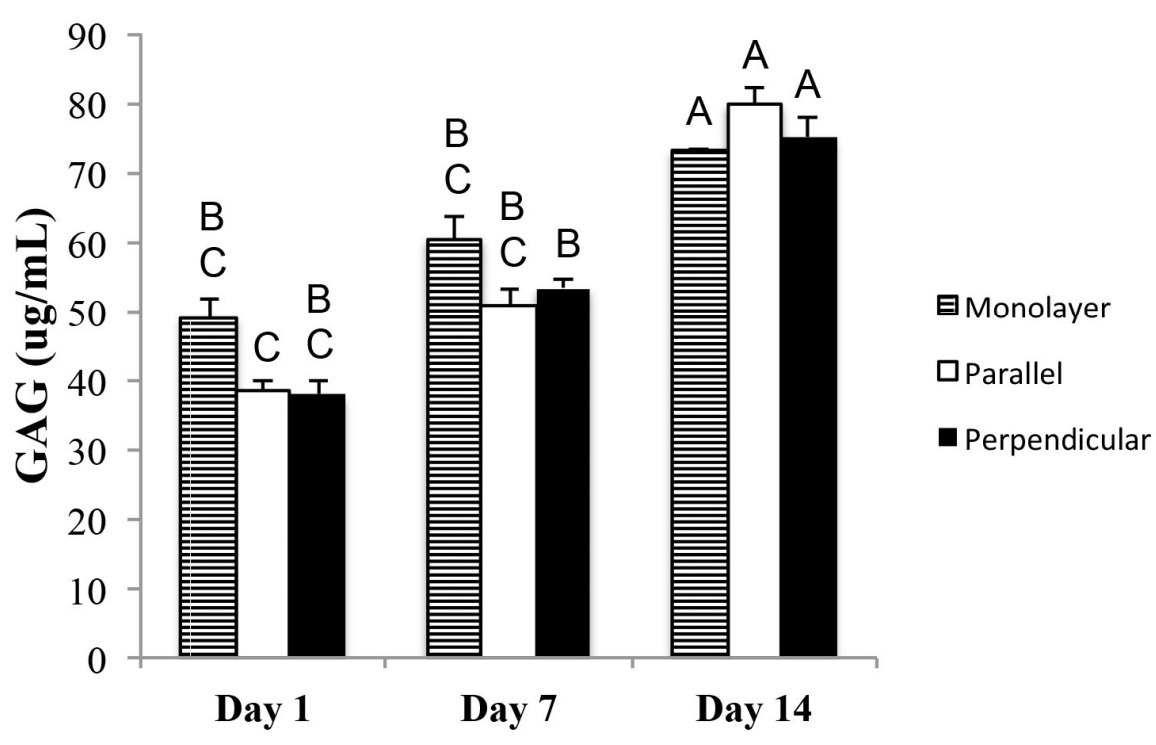

Figure 7. MSC production of the key matrix components. Collagen content quantification in media from cells in monolayer, aligned parallel scaffolds and aligned perpendicular scaffolds measured on days 1, 7, and 14 (A). $p=0.0038$ for increased collagen production between days 1, 7, and 14, regardless of fiber orientation; GAG content quantification in media from cells in monolayer, aligned parallel scaffolds and aligned perpendicular scaffolds measured on days 1, 7, and 14 (B). $p=0.0038$ for increased GAG production between all three days and fiber orientation. $p$-value $\leq 0.05$ were considered significant. Groups not connected by the same letter are significantly different. Error bars represent SEM.

\section{Discussion}

Aligned parallel and perpendicular suspended fiber networks were used to investigate cell response to microenvironmental cues of orientation and alignment; specifically tenogenic differentiation of equine bone marrow-derived MSCs on 3D nanofibers. We found that MSCs adhered to and elongated on fibrous scaffolds of both parallel and perpendicular oriented fibers, maintained viability, and migrated along both individual and multiple fibers regardless of fiber orientation. Previous studies using aligned fibrous scaffolds have shown that MSCs elongate along aligned fibrous scaffolds to promote tenocyte-like morphologies and tendon differentiation, while random electrospun fibrous scaffolds promote polygonal cell morphology and random cell orientation [16,31,32]. Instead of randomly oriented fibers, we used aligned perpendicular fibers resulting in primarily two different morphologies. These findings show that alignment cues affected cell morphology and cell alignment, providing preliminary evidence of tenogenesis.

Further evidence of tenogenesis was demonstrated by an increasing trend of SCX and COL I gene expression, in response to fiber orientation from both parallel and perpendicular fibers, over time. Topographical cues from the fibers control cytoskeletal rearrangement and result in changes in cell morphology that contribute towards differentiation [33-35]. Using aligned rope-like silk scaffolds, Maharam et al. have previously demonstrated the association between cell morphology and tendon differentiation with increased expression of COL I with increase in SCX [36]. However, it was unexpected to have similar changes in gene expression of MSCs from the parallel and perpendicular oriented fibers. This can be explained by the similarities in the cell-fiber interactions. The cells on both the parallel and perpendicular fibers interacted with the individual fibers due to fiber density. Previous studies have investigated tendon gene expression from cells cultured on fibrous scaffolds where tendon gene expression was not significantly altered, although this was a comparison between aligned 
and random scaffolds [37]. In another study, Gilchrist et al. compared aligned (parallel) and grid (perpendicular) architectural cues and observed an increase of gene expression of SCX and COL I from day 6 to day 12, with no significant differences in tendon gene expression between designs [22], similar to results observed in our scaffolds. Our findings suggest that alignment, rather than orientation, may be the predominant cue influencing tenogenic differentiation.

We investigated whether morphology and gene expression also correlated to the production of ECM components. We found equine MSCs produced increased amounts of collagen and GAGs over time in response to fibrous scaffolds, in accordance with previous studies. Although our study involved differences in fiber orientation compared to others, we found that the production of ECM components increased similarly. Moffat et al. showed an increase of collagen production from fibroblast like cells on aligned scaffolds compared to unaligned nanofiber scaffolds [38]. Therefore, increased collagen production was expected with both scaffold designs being highly aligned. We also found the GAG deposition to increase over time in all three groups from day 1, day 7 and day 14. An increase in proteogylcans may result in enhancement of fibril and fiber sliding, necessary for proper mechanical function of collagen in tendon [39]. These findings corroborate previous reports of elevated matrix production on nanofibers in response to both parallel and perpendicular orientation of fibers. Chainani et al. report a similar trend of increasing collagen and GAG content over a 28-day culture period [18]. Other studies report increased scaffold production over time, with no differences between aligned and nonaligned scaffolds [15]. Thus, our findings suggest that increased matrix production over time may not be due to fiber orientation, suggesting other factors including fiber diameter may be involved.

\section{Conclusions}

The aligned nanofiber scaffolds were manufactured with fiber characteristics mimicking native tendon fibrils; alignment and nano-sized fiber diameters. In addition, these scaffold provide cell infiltration, promote tendon-like gene expression, and matrix production. Topographical cues stimulated tenogenesis and emphasized the importance of alignment in determining cell fate. In addition, our study provides insight into the importance of optimizing scaffold design through adjustable parameters that influence MSC behavior and fate. As a whole, our findings suggest that nanofiber topography alone can influence tenogenic differentiation; specifically, fiber alignment promotes cell elongation and tendon-like characteristics of tendon-related markers and matrix production for MSC differentiation. Future studies will adjust scaffold parameters to further investigate how alignment cues affect MSC response and investigate molecular mechanisms involved in tendon differentiation. Our study demonstrated that aligned scaffolds induced changes in MSC morphology, gene expression, and scaffold production that support evidence of tenogenesis. Further exploration of fibrous scaffold parameters and MSC response in varying topographical environments may help to elucidate the optimal parameters needed to stimulate tenogenesis.

Supplementary Materials: The following are available online at http//www.mdpi.com/2076-3417/7/1/59/s1, Video S1: Mesenchymal stem cells on aligned parallel nanofibers, Video S2: Mesenchymal stem cells on aligned perpendicular nanofibers.

Acknowledgments: This study made use of Virginia Tech's Institute for Critical Technology and Applied Science and the Nanoscale Characterization and Fabrication Laboratory. The authors would like to acknowledge funding by the Stamps Family Charitable Foundation. A.S.N. would like to acknowledge partial support from NSF grant CMMI-1437101. The authors would like to thank Puja Sharma, Kevin Sheets, Daniel W. Youngstrom, Jade E. LaDow, and all other members of the STEP Laboratory and the Regenerative Medicine Research Laboratory.

Author Contributions: All authors conceived and designed the experiments; Tracee L. Popielarczyk performed the experiments; Tracee L. Popielarczyk and Jennifer G. Barrett analyzed the data; Amrinder S. Nain contributed reagents/materials/analysis tools; Tracee L. Popielarczyk wrote the paper.

Conflicts of Interest: The authors declare no conflict of interest. 


\section{References}

1. Spaas, J.H.; Guest, D.J.; Van de Walle, G.R. Tendon regeneration in human and equine athletes: Ubi Sumus-Quo Vadimus (where are we and where are we going to)? Sports Med. 2012, 42, 871-890. [CrossRef] [PubMed]

2. Webb, W.R.; Dale, T.P.; Lomas, A.J.; Zeng, G.D.; Wimpenny, I.; El Haj, A.J.; Forsyth, N.R.; Chen, G.Q. The application of poly(3-hydroxybutyrate-co-3-hydroxyhexanoate) scaffolds for tendon repair in the rat model. Biomaterials 2013, 34, 6683-6694. [CrossRef] [PubMed]

3. Randelli, P.; Conforti, E.; Piccoli, M.; Ragone, V.; Creo, P.; Cirillo, F.; Masuzzo, P.; Tringali, C.; Cabitza, P.; Tettamanti, G.; et al. Isolation and characterization of 2 new human rotator cuff and long head of biceps tendon cells possessing stem cell-like self-renewal and multipotential differentiation capacity. Am. J. Sport Med. 2013, 41, 1653-1664. [CrossRef] [PubMed]

4. Mazzocca, A.D.; McCarthy, M.B.R.; Chowaniec, D.; Cote, M.P.; Judson, C.H.; Apostolakos, J.; Solovyova, O.; Beitzel, K.; Arciero, R.A. Bone marrow-derived mesenchymal stem cells obtained during arthroscopic rotator cuff repair surgery show potential for tendon cell differentiation after treatment with insulin. Arthroscopy 2011, 27, 1459-1471. [CrossRef] [PubMed]

5. Czaplewski, S.K.; Tsai, T.-L.; Duenwald-Kuehl, S.E.; Vanderby, R., Jr.; Li, W.-J. Tenogenic differentiation of human induced pluripotent stem cell-derived mesenchymal stem cells dictated by properties of braided submicron fibrous scaffolds. Biomaterials 2014, 35, 6907-6917. [CrossRef] [PubMed]

6. Leung, M.; Jana, S.; Tsao, C.T.; Zhang, M.Q. Tenogenic differentiation of human bone marrow stem cells via a combinatory effect of aligned chitosan-polycaprolactone nanofibers and TGF-beta 3. J. Mater. Chem. B 2013, 1, 6516-6524. [CrossRef]

7. Jones, A.J.; Bee, J.A. Age-related and position-related heterogeneity of equine tendon extracellular-matrix composition. Res. Vet. Sci. 1990, 48, 357-364. [PubMed]

8. Lin, Y.L.; Brama, P.A.J.; Kiers, G.H.; van Weeren, P.R.; DeGroot, J. Extracellular matrix compositon of the equine superficial digital flexor tendon: Relationship with age and anatomical site. J. Vet. Med. A 2005, 52, 333-338. [CrossRef] [PubMed]

9. Liu, H.H.; Zhu, S.A.; Zhang, C.; Lu, P.; Hu, J.J.; Yin, Z.; Ma, Y.; Chen, X.; OuYang, H.W. Crucial transcription factors in tendon development and differentiation: Their potential for tendon regeneration. Cell Tissue Res. 2014, 356, 287-298. [CrossRef] [PubMed]

10. Murchison, N.D.; Price, B.A.; Conner, D.A.; Keene, D.R.; Olson, E.N.; Tabin, C.J.; Schweitzer, R. Regulation of tendon differentiation by scleraxis distinguishes force-transmitting tendons from muscle-anchoring tendons. Development 2007, 134, 2697-2708. [CrossRef] [PubMed]

11. Reed, S.A.; Johnson, S.E. Expression of scleraxis and tenascin C in equine adipose and umbilical cord blood derived stem cells is dependent upon substrata and FGF supplementation. Cytotechnology 2014, 66, 27-35. [CrossRef] [PubMed]

12. Birch, H.L.; Bailey, J.V.B.; Bailey, A.J.; Goodship, A.E. Age-related changes to the molecular and cellular components of equine flexor tendons. Equine Vet. J. 1999, 31, 391-396. [CrossRef] [PubMed]

13. Ryan, C.N.; Sorushanova, A.; Lomas, A.J.; Mullen, A.M.; Pandit, A.; Zeugolis, D.I. Glycosaminoglycans in tendon physiology, pathophysiology, and therapy. Bioconj. Chem. 2015, 26, 1237-1251. [CrossRef] [PubMed]

14. Kim, S.J.; Jang, D.H.; Park, W.H.; Min, B.M. Fabrication and characterization of 3-dimensional PLGA nanofiber/microfiber composite scaffolds. Polymer 2010, 51, 1320-1327. [CrossRef]

15. Orr, S.B.; Chainani, A.; Hippensteel, K.J.; Kishan, A.; Gilchrist, C.; Garrigues, N.W.; Ruch, D.S.; Guilak, F.; Little, D. Aligned multilayered electrospun scaffolds for rotator cuff tendon tissue engineering. Acta Biomater. 2015, 24, 117-126. [CrossRef] [PubMed]

16. Yin, Z.; Chen, X.; Chen, J.L.; Shen, W.L.; Nguyen, T.M.H.; Gao, L.; Ouyang, H.W. The regulation of tendon stem cell differentiation by the alignment of nanofibers. Biomaterials 2010, 31, 2163-2175. [CrossRef] [PubMed] 
17. Jiang, X.; Nai, M.H.; Lim, C.T.; Le Visage, C.; Chan, J.K.Y.; Chew, S.Y. Polysaccharide nanofibers with variable compliance for directing cell fate. J. Biomed. Mater. Res. Part A 2015, 103, 959-968. [CrossRef] [PubMed]

18. Chainani, A.; Hippensteel, K.J.; Kishan, A.; Garrigues, N.W.; Ruch, D.S.; Guilak, F.; Little, D. Multilayered electrospun scaffolds for tendon tissue engineering. Tissue Eng. Part A 2013, 19, 2594-2604. [CrossRef] [PubMed]

19. Chen, R.; Huang, C.; Ke, Q.F.; He, C.L.; Wang, H.S.; Mo, X.M. Preparation and characterization of coaxial electrospun thermoplastic polyurethane/collagen compound nanofibers for tissue engineering applications. Colloid Surf. B 2010, 79, 315-325. [CrossRef] [PubMed]

20. Kazanci, M. Solvent and temperature effects on folding of electrospun collagen nanofibers. Mater. Lett. 2014, 130, 223-226. [CrossRef]

21. Zhong, S.P.; Teo, W.E.; Zhu, X.; Beuerman, R.W.; Ramakrishna, S.; Yung, L.Y.L. An aligned nanofibrous collagen scaffold by electrospinning and its effects on in vitro fibroblast culture. J. Biomed. Mater. Res. Part A 2006, 79A, 456-463. [CrossRef] [PubMed]

22. Gilchrist, C.L.; Ruch, D.S.; Little, D.; Guilak, F. Micro-scale and meso-scale architectural cues cooperate and compete to direct aligned tissue formation. Biomaterials 2014, 35, 10015-10024. [CrossRef] [PubMed]

23. Kishore, V.; Bullock, W.; Sun, X.H.; Van Dyke, W.S.; Akkus, O. Tenogenic differentiation of human MSCs induced by the topography of electrochemically aligned collagen threads. Biomaterials 2012, 33, 2137-2144. [CrossRef] [PubMed]

24. Nain, A.S.; Sitti, M.; Jacobson, A.; Kowalewski, T.; Amon, C. Dry spinning based spinneret based tunable engineered parameters (STEP) technique for controlled and aligned deposition of polymeric nanofibers. Macromol. Rapid Commun. 2009, 30, 1406-1412. [CrossRef] [PubMed]

25. Sheets, K.; Wang, J.; Meehan, S.; Sharma, P.; Ng, C.; Khan, M.; Koons, B.; Behkam, B.; Nain, A.S. Cell-fiber interactions on aligned and suspended nanofiber scaffolds. J. Biomater. Tissue Eng. 2013, 3, 355-368. [CrossRef]

26. Wang, J.; Nain, A.S. Suspended micro/nanofiber hierarchical biological scaffolds fabricated using non-electrospinning STEP technique. Langmuir 2014, 30, 13641-13649. [CrossRef] [PubMed]

27. Schindelin, J.; Arganda-Carreras, I.; Frise, E.; Kaynig, V.; Longair, M.; Pietzsch, T.; Preibisch, S.; Rueden, C.; Saalfeld, S.; Schmid, B.; et al. Fiji: An open-source platform for biological-image analysis. Nat. Meth. 2012, 9, 676-682. [CrossRef] [PubMed]

28. Stewart, A.A.; Barrett, J.G.; Byron, C.R.; Yates, A.C.; Durgarn, S.S.; Evans, R.B.; Stewart, M.C. Comparison of equine tendon-, muscle-, and bone marrow-derived cells cultured on tendon matrix. Am. J. Vet. Res. 2009, 70, 750-757. [CrossRef] [PubMed]

29. Farndale, R.W.; Buttle, D.J.; Barrett, A.J. Improved quantitation and discrimination of sulphated glycosaminoglycans by use of dimethylmethylene blue. Biochim. Biophys. Acta (BBA) Gen. Subj. 1986, 883, 173-177. [CrossRef]

30. Voleti, P.B.; Buckley, M.R.; Soslowsky, L.J. Tendon healing: Repair and regeneration. Annu. Rev. Biomed. Eng. 2012, 14, 47-71. [CrossRef] [PubMed]

31. Youngstrom, D.W.; Barrett, J.G.; Jose, R.R.; Kaplan, D.L. Functional characterization of detergent-decellularized equine tendon extracellular matrix for tissue engineering applications. PLoS ONE 2013, 8, e64151. [CrossRef] [PubMed]

32. Younesi, M.; Islam, A.; Kishore, V.; Anderson, J.M.; Akkus, O. Tenogenic induction of human MSCs by anisotropically aligned collagen biotextiles. Adv. Funct. Mater. 2014, 24, 5762-5770. [CrossRef] [PubMed]

33. McBeath, R.; Pirone, D.M.; Nelson, C.M.; Bhadriraju, K.; Chen, C.S. Cell shape, cytoskeletal tension, and RhoA regulate stem cell lineage commitment. Dev. Cell 2004, 6, 483-495. [CrossRef]

34. Miyoshi, H.; Adachi, T. Topography design concept of a tissue engineering scaffold for controlling cell function and fate through actin cytoskeletal modulation. Tissue Eng. 2014, 20, 609-627. [CrossRef] [PubMed]

35. Yin, Z.; Chen, X.; Song, H.-X.; Hu, J.-J.; Tang, Q.-M.; Zhu, T.; Shen, W.-L.; Chen, J.-L.; Liu, H.; Heng, B.C.; et al. Electrospun scaffolds for multiple tissues regeneration in vivo through topography dependent induction of lineage specific differentiation. Biomaterials 2015, 44, 173-185. [CrossRef] [PubMed] 
36. Maharam, E.; Yaport, M.; Villanueva, N.L.; Akinyibi, T.; Laudier, D.; He, Z.; Leong, D.J.; Sun, H.B. Rho/Rock signal transduction pathway is required for MSC tenogenic differentiation. Bone Res. 2015, 3, 15015. [CrossRef] [PubMed]

37. Subramony, S.D.; Dargis, B.R.; Castillo, M.; Azeloglu, E.U.; Tracey, M.S.; Su, A.; Lu, H.H. The guidance of stem cell differentiation by substrate alignment and mechanical stimulation. Biomaterials 2013, 34, 1942-1953. [CrossRef] [PubMed]

38. Moffat, K.L.; Kwei, A.S.-P.; Spalazzi, J.P.; Doty, S.B.; Levine, W.N.; Lu, H.H. Novel nanofiber-based scaffold for rotator cuff repair and augmentation. Tissue Eng. Part A 2008, 15, 115-126. [CrossRef] [PubMed]

39. Franchi, M.; Torricelli, P.; Giavaresi, G.; Fini, M. Role of moderate exercising on Achilles tendon collagen crimping patterns and proteoglycans. Connect. Tissue Res. 2013, 54, 267-274. [CrossRef] [PubMed]

(C) 2017 by the authors; licensee MDPI, Basel, Switzerland. This article is an open access article distributed under the terms and conditions of the Creative Commons Attribution (CC-BY) license (http:/ / creativecommons.org/licenses/by/4.0/). 